Hell u. v. Günthert: Beitr. z. Kenntn. des Anethols. 193

\title{
Beiträge zur Kenntniss des Anethols.
}

\section{Mittheilung. ${ }^{1}$ )}

\section{Ueber die Einwirkung des Broms anf Anethol; von}

\section{Hell und O. von Günthert.}

Bei der Darstellung des gebromten Ketons

$\mathrm{C}_{6} \mathrm{H}_{3} \mathrm{Br}\left(\mathrm{OCH}_{3}\right) \cdot \mathrm{CHBr}$. $\mathrm{CO} . \mathrm{CH}_{3}$ oder

$\mathrm{C}_{8} \mathrm{H}_{3} \mathrm{Br}\left(\mathrm{OCH}_{3}\right) \mathrm{CO}$. $\mathrm{CHBrCH}_{3}$

aus dem Monobromanetholdibromid mittelst einer Auflösung von Chromsäure in Eisessig wurde häufig neben den aus heissem Alkohol sich abscheidenden Krystallen des Ketons auch noch eine viel leichter lösliche Verbindung erhalten, welche aus den Mutterlaugen in derben, stark durchsichtigen Krystallen sich absetzte, und einen geringeren Bromgehalt besass. Diese Verbindung entstand namentlich dann in grösserer Menge, wenn das Produkt der Einwirkung von Brom auf Anethol nur durch Waschen mit kaltem Alkohol gereinigt, nicht aber wiederholt aus Petroleumäther umkrystallisirt wurde. Es lag daher nahe zu vermuthen, dass dieses Produkt der Oxydation des A netholdibromids, das schon von Ladenburg dargestellt und untersucht wurde, seine Entstehung verdanke, und es schien interessant, die Untersuchung sowohl auf bromärmere als auch bromreichere Derivate des Anethols auszudehnen.

Ausserdem hatten die Untersuchungen von Gärttner noch manche Lücken offen gelassen, die bei der anscheinend grossen Reactionsfähigkeit des Bromanetholdibromids, wie namentlich des daraus dargestellten Ketons durch weitergehende Versuche auszufüllen waren.

Als Ausgangsmaterial diente das Sternanisöl, das fast ganz aus krystallisirbarem Anethol besteht, so dass durch Absaugen nur ganz wenig von flüssigen Beimengungen abgetrennt werden konnten. Bei der fractionirten Destillation zeigten jedoch die durch Absaugen gereinigten Anetholkrystalle keineswegs einen.

1) 1. Mitth. s. dies. Journ. [2] 51, 422.

Journal f. prakt. Chemie [2] Bd. 52. 
194 Hell u. v. Günthert: Beitr. z. Kenntn. des Anethols.

constanten Siedepunkt. Die Hauptmasse destillirte zwischen $226^{\circ}-236^{\circ}$ über, und diese wurde zu den folgenden Versuchen verwendet, ein kleiner Theil siedete jedoch auch niedriger, und bei Verarbeitung mehrerer Kilogramm Sternanisöl konnte eine etwa $150 \mathrm{Grm}$. betragende Menge vom Siedep. $210^{\circ}-220^{\circ}$ und etwa die doppelte Menge rom Siedep. 220 $228^{\circ}$ abgesondert werden.

Zur Darstellung des Monobromanetholdibromids diente das schon von $G$ ärttner angewandte Verfahren. Das Anethol in Mengen von etwa $20 \mathrm{Grm}$. wurde in der 2-3 fachen Menge absoluten Aethers, welcher vorher durch Waschen mit Wasser, Trocknen über Chlorcalcium und Rectification über metallischem Natrium vollständig alkohol- und wasserfrei gemacht war, gelöst, und nun mittelst eines Tropftrichters das Brom anfänglich sehr langsam und unter Abkühlung zugebracht. Die Einwirkung ist eine sehr energische, das eintröpfelnde Brom verschwindet unter starkem Zischen sofort, und wenn man nicht von Zeit zu Zeit den Zulauf des Broms unterbricht, so gelangt der Aether trotz äusserer Abkühlung in wallendes Sieden. Ist alles Brom, man wendet etwas mehr als die theoretische Menge an, eingetragen, so lässt man am besten durch Eingiessen in flache Schalen und Stehen an der Luft den Aether verdunsten, und reinigt das zurückgebliebene, gewöhnlich durch freies Brom noch braungefärbte Produkt durch Anreiben und Waschen mit kaltem Alkohol und krystallisirt die durch diese Behandlung schon ganz weiss gewordene Masse nach dem Trocknen aus schwer flüchtigem Petroleumäther einbis zweimal um. Man erhält so das Monobromanetholdibromid ganz rein, in Form ziemlich dicker, stark glänzender Nadeln vom Schmelzp. $108^{\circ}$.

Die Brom bestimmung ergab:

0,245 Grm. Substanz gaben 0,356 Grm. $\mathrm{AgBr}=61,63 \% \mathrm{Br}$ statt $62,0 \%$, welche die Formel $\mathrm{C}_{10} \mathrm{H}_{11} \mathrm{Br}_{3} \mathrm{O}$ verlangt.

Nach Gärttner und Hell ist die Einwirkung von heissem Alkohol zu vermeiden, weil dadurch Bildung schwer krystallisirbarer Produkte stattfindet. Es war von Interesse, den Grund dieses Verhaltens näher kennen zu lernen und die Natur der hierbei entstehenden Verbindung aufzuklären. 
Hell u. v. Günthert: Beitr. z. Kenntn. des Anethols. 195

Einwirkung von kochendem Aethylalkohol auf

Monobromanetholdibromid.

50 Grm. des Bromids wurden mit absolutem Alkohol einige Tage lang am Rückflusskühler gekocht. Nach dem $A b$ destilliren des Alkohols, welcher den deutlichen Geruch nach Bromäthyl zeigte, hinterblieb eine syrupdicke Masse, welche in Wasser gegossen, nach einiger Zeit erstarrte, und aus Aether umkrystallisirt werden konnte. Man erhielt so schöne, lange, prismatische Krystalle, welche geruch- und geschmacklos waren und schon bei $62^{\circ}$ schmolzen. In Aether, Alkohol, Benzol und Chloroform waren dieselben leicht, in Petroleumäther dagegen schwer löslich.

Zwei Brombestimmungen ergaben übereinstimmende Resultate.

0,215 Grm. Substanz gaben 0,265 Grm. AgBr oder 52,0\% Br.

$0,228 \mathrm{Grm}$. Substanz gaben 0,280 Grm. AgBr oder 52,19\% Br.

Die Formel $\mathrm{C}_{10} \mathrm{H}_{10} \mathrm{Br}_{2} \mathrm{O}$ verlangt: $52,28 \% \mathrm{Br}$.

Die Einwirkung des absoluten Alkohols findet in der Weise statt, dass aus dem Monobromanetholdibromid $1 \mathrm{Mol}$. $\mathrm{HBr}$ austritt.

Einwirkung von Wasser auf das Bromid.

Auch Wasser wirkt bei höherer Temperatur verändernd auf das Monobromanetholdibromid ein, nur ist diese Einwirkung eine etwas andere.

Werden $50 \mathrm{Grm}$. des Bromids mit viel Wasser mehrere Tage lang am Rückflusskühler gekocht, so resultirt eine braune, zähe Masse, die auch nach wochen- und monatelangem Stehen nicht erstarrte und auch aus Aether, Alkohol oder Benzol, in welchen Lösungsmitteln sie leicht löslich war, nicht krystallisirt werden konnte. Längere Zeit über Schwefelsäure getrocknet, ergab sie bei der Bestimmung ibres Bromgehalts folgende Zahlen :

0,260 Grm. Substanz gaben 0,302 Grm. AgBr oder 49,2\% Br.

0,275 Grm. Substanz gaben 0,320 Grm. AgBr oder 49,45\% Br.

Wenn man trotz der keine Garantien für die Reinheit bietenden Beschaffenheit des Produkts dem Resultat der Brombestimmung einigen Werth beilegen will, so würde sich ergeben, dass die Einwirkung des Wassers nicht in einer Abspaltung von Bromwasserstoff, sondern in einer Substitution von Brom 
196 Hell u. v. Günthert: Beitr. z. Kenntn. des Anethols.

durch Hydroxyl bestanden hätte, denn die Formel $\mathrm{O}_{10} \mathrm{~B}_{12} \mathrm{Br}_{2} \mathrm{O}_{2}$, d. h. $\mathrm{C}_{10} \mathrm{H}_{11} \mathrm{Br}_{3} \mathrm{O}+\mathrm{H}_{2} \mathrm{O}=\mathrm{C}_{10} \mathrm{H}_{11} \mathrm{Br}_{2} \mathrm{O}(\mathrm{OH})+\mathrm{HBr}$ verlangt $49,3 \%$ Brom.

Um zu erfahren, an welcher Stelle der Austausch des Broms gegen $\mathrm{OH}$ stattgefunden habe, wurde das Produkt einer Oxydation mit Chromsäure und Eisessig unterworfen, welche schon in der Kälte deutlich unter schwacher Bromentwicklung von statten ging und namentlich beim Erwärmen zu einer energischen Reaction sich steigerte. Nach dem Abdestilliren des Eisessigs fiel auf Wasserzusatz eine braune, gallertartige Masse von scharfem, die Augen heftig reizenden Geruch aus, aus welcher durch Natriumcarbonat etwas Bromanissäure ausgezogen werden konnte. Die Hauptmenge blieb aber ungelöst. Dieselbe konnte aber nicht fest und rein erhalten werden, es unterblieb daher eine nähere Untersuchung. Möglicher Weise besteht die Verbindung aus einem acroleïnartigen Oxydationsprodukte des Carbinols. Eine weitere Untersuchung dieses Produkts steht in Aussicht.

Einwirkung von Anilin auf Monobromanetholbromid und auf das daraus durch Oxydation entstehende Keton.

Bemerkenswerth ist ferner die Einwirkung des Anilins auf das Monobromanetholdibromid. Beim Auflösen des letzteren in überschüssigem Anilin fand unter lebhafter Reaction die Ausscheidung von bromwasserstoffsaurem Anilin statt, und wach dem Abfiltriren der Anilinlösung und Versetzen mit Salzsäure bleibt ein gelb gefürbtes Pulver zurück, welches bei $7^{\circ}$ schmilzt, sich ziemlich leicht in Aether, Alkohol und Benzol löst, nach dem Verdunsten der Lösungsmittel aber als harzige Masse zurückbleibt und beim Versetzen mit Chromsäure in eine tiefschwarz gefärbte Verbindung übergeht.

Bei der Brombestimmung gaben:

0,261 Grm. Substanz 0,161 Grm. AgBr oder 26,05\% Br.

Die Formel $\mathrm{C}_{10} \mathrm{H}_{10} \mathrm{OBrN}$ verlangt 25,15\% Br.

Die Oxydation mit Kaliumpermanganat findet gleichfalls unter lebhafter Reaction statt, es entwickelt sich Anilin und aus der alkalischen Lösung scheidet Schwefelsäure einen voluminösen Niederschlag $a b$, der nach wiederholtem Lösen in 
Alkalien und Wiederabscheiden durch Säuren bei $213^{\circ}$ schmolz, in Alkohol und Aether leicht löslich war und aus letzterem beim Verdunsten in derben Krystallen sich abschied. Dem Schmelzpunkte nach ist dieses Oxydationsprodukt Monobromanissäure $\mathrm{C}_{8} \mathrm{H}_{3}\left(\mathrm{BrOCH}_{3}\right) \mathrm{COOH}$.

Die Einwirkung des Anilins auf Monobromanetholdibromid scheint darnach entsprechend der Gleichung:

$$
\mathrm{C}_{10} \mathrm{H}_{11} \mathrm{Br}_{3} \mathrm{O}+3 \mathrm{C}_{8} \mathrm{H}_{5} \mathrm{NH}_{2}=\mathrm{C}_{18} \mathrm{H}_{16} \mathrm{BrON}+2 \mathrm{C}_{6} \mathrm{H}_{5} \mathrm{NH}_{2} \mathrm{HBr}
$$

vor sich gegangen $\mathrm{zu}$ sein.

Ein besser charakterisirtes Derivat war bei der Einwirkung des Anilins auf das gebromte Keton zu erwarten, welches, wie schon Gärttner und Hell zeigten, durch Ammoniak in das leicht rein $\mathrm{zu}$ erhaltende isoindolartige Condensationsprodukt übergeführt werden konnte.

$\mathrm{Za}$ diesem $\mathrm{Z}$ weck wurde eine grössere Menge des Monobromanetholdibromids mit Chromsäure in eisessigsaurer Lösung oxydirt. Wir nahmen diesmal die Oxydation in offener Schale und unter Anwendung von weniger Eisessig vor. Dieselbe verlief unter diesen Umständen sehr viel rascher, wenn auch heftiger, und das entstandene Keton krystallisirte sofort beim Erkalten aus der Chromacetatlösung in feinen Nadeln heraus, die durch Auswaschen mit Wasser auf dem Saugfilter fast ganz rein erhalten werden konnten.

Nach dem Auskochen mit Natriumcarbonat wurde die Verbindung aus Alkohol umkrystallisirt. Der Schmelzpunkt lag bei $99^{\circ}$.

Die Brombestimmung ergab:

$0,290 \mathrm{Grm}$. Substanz lieferten $0,340 \mathrm{Grm}$. AgBr oder $49,65 \% \mathrm{Br}$. Berechnet für $\mathrm{C}_{10} \mathrm{H}_{10} \mathrm{Br}_{2} \mathrm{O}_{2} 49,68 \% \mathrm{Br}$.

Beim Erhitzen dieses Ketons mit überschüssigem Anilin über der freien Flamme schied sich bromwasserstoffsaures Anilin aus, welches abfiltrirt wurde, während die dunkelroth gefärbte Lösung zur Entfernung des überschüssigen Anilins mit Salzsäure behandelt wurde. Es bildete sich hierbei zuerst ein harzig ausfallender Körper, welcher aber nach einiger Zeit fest und pulverig wurde. Er wurde zuerst wiederholt mit Salzsäure ausgewaschen und dann nach dem Trocknen an der Luft in Alkohol gelöst. Aus dieser Lösung schieden sich beim 
198 Hell u. v.Günthert: Beitr. z. Kenntn. des Anethols.

Verdunsten neben einem dunkelrothen Harze feine, gelblich gefărbte, meistens zu kleinen Büscheln vereinigte Nadeln ab, welche durch Abschlemmen von dem an den Wänden festhaftenden Harze getrennt und durch wiederholtes Umkrystallisiren aus Alkohol von dem constanten Schmelzp. $119^{\circ}$ erhalten werden konnten.

Die Brombestimmung ergab für:

0,304 Grm. Substanz 0,178 Grm. AgBr oder 24,68\% Br.

Die Formel $\mathrm{C}_{16} \mathrm{H}_{16} \mathrm{O}_{2} \mathrm{BrN}$ verlangt $23,95 \% \mathrm{Br}$.

Bei der Elementaranalyse wurde erhalten aus:

0,301 Grm. Substanz 0,635 Grm. $\mathrm{CO}_{2}$ und 0,127 Grm. $\mathrm{H}_{2} \mathrm{O}$.

\begin{tabular}{|c|c|}
\hline Berechnet: & Gefunden: \\
\hline 57,48 & $57,47 \%$ \\
\hline 4,79 & 4,68, \\
\hline
\end{tabular}

Die neue Verbindung ist darnach einfach durch Eintritt des Anilinrestes an Stelle des Bromatoms der Seitenkette entstanden. Sie löst sich leicht in Aether, Alkohol, Benzol und Chloroform, dagegen schwer in Petroleumäther. In conc. Schwefelsäure löst sie sich ebenfalls mit grünlicher Färbung und fällt auf Zusatz von Wasser wieder unverändert aus.

Analoge Untersuchungen mit Anetholdibromid.

Lässt man Brom im Ueberschusse in das mit Aether verdünnte Anethol eintröpfeln, so resultirt fast ausschliesslich das schon beschriebene Monobromanetholdibromid. Wendet man aber auf $1 \mathrm{Mol}$. Anethol genau $1 \mathrm{Mol}$. Brom an, und sorgt überdies dafür, dass durch Anwendung von kleineren Portionen, höchstens $20 \mathrm{Grm}$., Anethol, durch Verdünnen mit dem 2-3fachen Volumen reinen Aethers und durch starke Abkühlung mit Eiswasser das langsam eintröpfelnde Brom keine erhebliche Temperatursteigerung hervorbringen kann, so besteht die nach dem Verdunsten des Aethers zurückbleibende krystallinische Masse, welche durch Anreiben mit kaltem Petroleumäther und Abpressen zwischen Papier, schliesslich durch wiederholtes Umkrystallisiren aus heissem leicht flüchtigem Petroleumäther ganz rein erhalten werden kann, aus dem schon von Ladenburg dargestellten Anetholdibromid. Die feinen, nadelförmigen Krystalle schmelzen bei $67^{\circ}$ und stimmen darin mit den Angaben von Ladenburg überein. 
Die Brombestimmung führte zu folgenden Zahlen:

$0,228 \mathrm{Grm}$. Substanz gaben $0,278 \mathrm{Grm}$. AgBr oder $51,75 \% \mathrm{Br}$.

Die Formel $\mathrm{C}_{10} \mathrm{H}_{12} \mathrm{Br}_{2} \mathrm{O}$ verlangt $51,9 \% \mathrm{Br}$.

Aus der dunklen, schmierigen Mutterlauge, welche beim Umkrystallisiren zurückblieb, wurden bei längerem Stehen noch weitere Krystalle gewonnen, welche das Aussehen des Monobromanetholdibromids $\mathrm{C}_{10} \mathrm{H}_{11} \mathrm{Br}_{3} \mathrm{O}$ besassen und durch ihren Schmelzp. $108^{\circ}$ sich als solches erwiesen. Es geht daraus hervor, dass auch bei der grössten Vorsicht sich die Bildung des Monobromanetholdibromids kaum vermeiden lässt.

Das Anetholdibromid verhält sich im Allgemeinen ganz analog wie das einfach bromirte Produkt, doch konnten auch einige bemerkenswerthe Unterschiede nachgewiesen werden.

\section{Einwirkung von Aethylalkohol auf das Anethol-} dibromid.

Kochender Alkohol verändert das Dibromid ebenfalls. Lässt man $20 \mathrm{Grm}$. des Anetholdibromids mit absolutem Alkohol am Rückflusskühler einige Tage kochen, so bleibt nach dem Abdestilliren des Alkohols eine dunkelbraun gefärbte harzige Masse zurück, welche einen starken Anetholgeruch besitzt und trotz aller Bemühungen nicht in festem Zustande erhalten werden konnte. Sie löste sich leicht in Aether, Alkohol, Benzol, Chloroform und Eisessig, theilweise auch in Petroleumäther. Beim Verdunsten der Lösungsmittel aber blieb die gleiche dunkle Schmiere zurück.

Die Brombestimmung ergab:

$0,210 \mathrm{Grm}$. Substanz lieferten 0,142 $\mathrm{Grm}$. AgBr oder $28,57 \% \mathrm{Br}$, während die nach Analogie bei dem Monobromanetholdibromid entstandene Verbindung $\mathrm{C}_{10} \mathrm{H}_{11} \mathrm{BrO} 35,2 \% \mathrm{Br}$, und die dureh den Eintritt von $\mathrm{OC}_{2} \mathrm{H}_{5}$ an Stelle von Brom gebildete Verbindung $\mathrm{C}_{12} \mathrm{H}_{17} \mathrm{BrO} \mathrm{O}_{2} 29,3 \% \mathrm{Br}$ verlangt.

Es scheint danach, dass die Umsetzung eher in letzterer Weise stattgefunden habe, wir müssen uns jedoch bei der Unmöglichkeit, die Verbindung bis jetzt in reinem Zustande zu erhalten, eine bestimmtere Ansicht bis später vorbehalten.

Oxydation des Anetholdibromids mit Chromsäure.

Bei der Oxydation des Dibromids mit Chromsäure in Eisessig tritt schon in der Kälte, unter Entwicklung von Brom, 
eine lebhafte Reaction ein. Nachdem durch Erwärmen die Oxydation zu Ende gebracht worden war, und die Lösung eine rein dunkelgrüne Färbung angenommen hatte, wurde der Eisessig abdestillirt, und der Rückstand in Wasser gegossen. Es schied sich hierbei eine gelblich gefärbte, halbfeste Masse ab, welche durch Filtriren von der Chromacetatlösung getrennt, mit Wasser ausgewaschen und sodann mit Natriumcarbonat ausgekocht wurde. Aus heissem Petroleumäther konnte dann die Masse umkrystallisirt werden.

Die Trennung des aus dem Dibromid entstandenen Ketons von dem aus dem Monobromanetholdibromid entstehenden, dessen Bildung sich auch bei noch so vorsichtigem Bromiren des Anethols nicht vermeiden lässt, bietet einige Schwierigkeit. Am besten gelangt man zum Ziel, wenn man nach dem Erkalten der heissen Lösung die Mutterlauge von den ausgeschiedenen Krystallen abgiesst und langsam verdunsten lässt. In den beim Erkalten ausgeschiedenen Krystallen findet sich fast ausschliesslich das höher bromirte Keton vor, während in der beim Verdunsten erhaltenen Krystallmasse das einfach bromirte Keton $\mathrm{C}_{10} \mathrm{H}_{11} \mathrm{BrO}_{2}$ vorhanden ist, welches durch 5-6 malige Wiederholung dieser Operation schliesslich rein erhalten werden kann. Das Keton scheidet sich in schönen, grossen, stark lichtbrechenden, flächenreichen Krystallen ab, die fast denselben Schmelzpunkt wie das nicht oxydirte Anetholdibromid, nämlich $65^{\circ}-67^{\circ}$ besitzen. Die Verbindung besitzt einen eigenthümlich beissenden Geruch und erregte, auf die Haut gebracht, auf zarteren Theilen derselben, heftiges Brennen. Sie löst sich leicht in Alkohol, Aether und Petroleumäther, die Ausbeute an reinem Material ist jedoch gering. So wurden von 200 Grm. Anetholdibromids nur ca. 25 Grm. des Oxydationsproduktes erhalten.

Bei der Brombestimmung gaben:

0,200 Grm. Substanz 0,154 Grm. AgBr oder 32,50\% Br.

Berechnet für $\mathrm{C}_{10} \mathrm{H}_{11} \mathrm{BrO}_{2} \quad 32,9 \% \mathrm{Br}$.

Der Verlauf der Oxydation findet also hier in ähnlicher Weise wie bei dem Monobromanetholdibromid statt. 
Einwirkung von alkoholischem Ammoniak auf das Keton.

Beim Erhitzen mit überschüssigem alkoholischem Ammoniak im Wasserbade löste sich dasselbe unter Gelbfärbung auf, und nach dem Abdestilliren des Alkohols und Ammoniaks bleibt eine grïnliche Masse zurück, die in Wasser unlöslich ist und damit ausgewaschen werden kann. In heissem Alkohol gelöst, scheidet sich beim Erkalten ein gelbes Krystallpulver rom Schmelzp. $176^{\circ}$ ab. Dieses löst sich leicht in Aether, schwerer in Alkohol mit intensiv goldgelber Farbe, in Petroleumäther ist es unlöslich.

Eine vollständige Analyse ergab folgende Zahlen:

$0,263 \mathrm{Grm}$. Substanz lieferten bei $21^{\circ}$ und $733 \mathrm{Mm}$. 20,0 Ccm. N.

$0,339 \mathrm{Grm}$. Substanz lieferten $0,921 \mathrm{Grm} . \mathrm{CO}_{2}$ und $0,179 \mathrm{Grm} . \mathrm{H}_{2} \mathrm{O}$.

Berechnet für $\mathrm{O}_{10} \mathrm{H}_{11} \mathrm{NO}$ :

$\begin{array}{ccc}\mathrm{C} & \mathbf{7 4 , 5} & \mathbf{7 4 , 0} \% \\ \mathrm{H} & 6,8 & \mathbf{5 , 8 6}, \\ \mathrm{N} & 8,6 & 8,5 \% .\end{array}$

Die Einwirkung des Ammoniaks hat danach in derselben Weise stattgefunden, wie bei dem zweifach gebromten Keton $\mathrm{C}_{10} \mathrm{H}_{10} \mathrm{Br}_{2} \mathrm{O}_{2}$.

Zuerst wurde das Bromatom der Seitenkette durch $\mathrm{NH}_{2}$ ersetzt und dieses condensirt sich mit der Carbonylgruppe unter Austritt von $1 \mathrm{Mol}$. $\mathrm{H}_{2} \mathrm{O}$, unter Bildung eines isoindolartigen Körpers:

$\mathrm{C}_{8} \mathrm{H}_{4}\left(\mathrm{OCH}_{3}\right) \mathrm{CH}-\mathrm{C} . \mathrm{CH}_{3}$ oder $\mathrm{C}_{6} \mathrm{H}_{4}\left(\mathrm{OCH}_{3}\right) \mathrm{C}-\mathrm{CH} . \mathrm{CH}_{3}$.

Auch hier muss es dahin gestellt bleiben, ob der Verbindung das Mol.-Gew. $\mathrm{C}_{10} \mathrm{H}_{11} \mathrm{NO}$ oder ein doppelt so grosses zukommt.

Einwirkung von Anilin auf das Keton.

Beim Erhitzen mit Anilin in alkoholischer oder ätherischer Lösung bildet sich neben bromwasserstoffsaurem Anilin eine harzige Masse, welche sich in Aether, Alkohol, Benzol, Chloroform und Eisessig mit rothbrauner Farbe löste, beim Verdunstenlassen dieser Lösungsmittel aber immer wieder nur in Form eines dunklen Harzes erhalten werden konnte. Da alle 
202 Hell u. v.Günthert: Beitr. z. Kenntn. des Anethols.

Versuche scheiterten, ein krystallisirtes Produkt zu erhalten, auch die Einwirkung von Acetylchlorid zu keinem greifbaren Resultate führte, so wurde diese Einwirkung nicht näher verfolgt. Es ist jedoch wahrscheinlich, dass dieselbe in analoger Weise wie bei dem zweifach gebromten Keton stattgefunden hat, d. h. dass das Bromatom der Seitenkette durch den Anilinrest ersetzt wurde.

\section{Oxydation des Ketons mit Kaliumpermanganat.}

Oxydirt man das einfach gebromte Keton mit der 3 Atomen Sauerstoff entsprechenden Menge übermangansauren Kalis in wässriger Lösung, so erhält man aus der von dem Braunstein abfiltrirten alkalischen Lösung beim Versetzen mit Schwefelsäure eine reichliche Menge einer aus heissem Wasser in Nadeln krystallisirenden Säure, welche nach ihrem Schmelzpunkt $185^{\circ}$ aus reiner Anissäure bestand. Die von der Anissäure abfiltrirte saure Flüssigkeit wurde bis auf $1 / 3$ abdestillirt, das Destillat mit Silbercarbonat gesättigt, wodurch eine grosse Menge von Silberacetat erhalten werden konnte. Aus dem Rückstande der Destillation schied sich beim Erkalten noch ein kleiner Theil von Anissäure aus. Dieselbe wurde durch Filtriren entfernt und das Filtrat mit Aether ausgeschüttelt. Nach dem Verdunsten des Aethers konnte jedoch eine leicht lösliche Ketonsäure nicht nachgewiesen werden. Der unbedeutende Rückstand, der beim Verdunsten des Aethers hinterblieb, löste sich ziemlich schwer in Wasser und bestand, seinem Schmelzp. $185^{\circ}$ nach zu urtheilen, ausschliesslich aus Anissäure. Die $\mathrm{Zu}$ sammensetzung des Anetholdibromids muss nach diesen Resultaten durch die Formel $\mathrm{C}_{6} \mathrm{H}_{4}\left(\mathrm{OCH}_{3}\right)$. $\mathrm{CHBr}$. $\mathrm{CHBr} . \mathrm{CH}_{3}$ und das durch Oxydation daraus erbaltene Keton durch die Formel $\mathrm{C}_{6} \mathrm{H}_{4}\left(\mathrm{OCH}_{3}\right) \mathrm{CHBr}$. $\mathrm{COCH}_{3}$, bezw. $\mathrm{C}_{8} \mathrm{H}_{4}\left(\mathrm{OCH}_{3}\right) \mathrm{CO}$. $\mathrm{CHBr}^{-\mathrm{CH}_{3}}$ ausgedrückt werden.

Höbere Bromsubstitutionsprodukte des Anetholdibromids.

Wird das auf die oben beschriebene Weise dargestellte Monobromanetholdibromid mit $1 \mathrm{Mol}$. Brom $(3 \mathrm{Ccm}$. Brom auf 20 Grm. des Bromids) in Röhren eingeschmolzen und 
längere Zeit im Wasserbade erhitzt, so findet eine weitere Einwirkung des Broms statt; es bildet sich viel Bromwasserstoff, den man, um ein Zerspringen der Röhren zu vermeiden, von Zeit zu Zeit aus derselben entlässt, und ein syrupförmiger Räckstand, den man durch Auflösen in Aether und Verdunsten der Aetherlösung bei gewöhnlicher Temperatur theilweise krystallisirt erhalten kann. Der von einer syrupförmigen Mutterlauge durchtränkte Krystallbrei wird mit kaltem Alkohol angerieben, zwischen Filtrirpapier gepresst, zum zweiten $\mathrm{Mal}$ mit leicht flüchtigem Petroleumäther angerieben und auf dem Saugfilter damit ausgewaschen, wobei ein verhältnissmässig rein aussehendes Krystallmehl zurückblieb. Dasselbe wurde aus heissem Petroleumäther umkrystallisirt und so in feinen, schönen Nadeln vom Schmelzp. $113^{\circ}-114^{0}$ erhalten.

Zwei Brombestimmungen ergaben übereinstimmende Resultate:

$0,284 \mathrm{Grm}$. Substanz lieferten $0,460 \mathrm{Grm}$. AgBr oder $68,66 \% \mathrm{Br}$.

$0,282 \mathrm{Grm}$. Substanz lieferten 0,454 Grm. AgBr oder $68,43 \% \mathrm{Br}$.

Die Formel $\mathrm{C}_{10} \mathrm{H}_{10} \mathrm{Br}_{4} \mathrm{O}$ verlangt $68,6 \% \mathrm{Br}$.

Die Ausbeute an dem reinen Dibromanetholdibromid $\mathrm{C}_{10} \mathrm{H}_{10} \mathrm{Br}_{4} \mathrm{O}$ ist nicht sehr gross. Die Hauptmasse bildet eine tief dunkelbraune, syrupartige Mutterlauge, aus welcher nach längerem Stehen bei niederer Temperatur sich grosse, dicke Krystalle abschieden. Dieselben wurden durch Abpressen möglichst von der anhängenden Mutterlange befreit und aus leicht flüchtigem Petroleumäther umkrystallisirt, wobei sie warzenförmige Krystalle gaben, welche einen erheblich niedrigeren Schmelzpunkt, als die obigen feinen Nadeln, nämlich $89^{\circ}$ besassen.

Die Brombestimmung stimmte jedoch auch hier mit einem Dibromanetholdibromid, $\mathrm{C}_{10} \mathrm{H}_{10} \mathrm{Br}_{4} \mathrm{O}$, überein.

$0,304 \mathrm{Grm}$. Substanz gaben 0,490 Grm. AgBr oder $68,42 \% \mathrm{Br}$.

$0,300 \mathrm{Grm}$. Substanz gaben 0,484 Grm. AgBr oder $68,33 \% \mathrm{Br}$, so dass man hier zwei isomere Verbindungen anzunehmen hat. Diese beiden unterscheiden sich neben ihrem verschiedenen Schmelzpunkte auch noch durch die verschiedene Löslichkeit in Petroleumäther. Das bei $113^{\circ}-114^{\circ}$ schmelzende Produkt ist darin viel schwerer löslich und krystallisirt fast vollständig heraus, das bei $89^{\circ}$ schmelzende Produkt ist dagegen schon in kaltem Petroleumäther löslich und scheidet sich erst beim 
204 Hell u. v. Günthert: Beitr. z. Kenntn. des Anethols.

Verdunsten desselben aus. Bei der Oxydation mit Chromsäure in eisessigsaurer Lösung erhält man aus beiden Verbindungen, wio es scheint, dieselben Produkte.

\section{Reduction des Dibromanetholdibromids mit Zinkstaub.}

Brachte man in die ätherische Lösung des Tetrabromids überschüssigen Zinkstaub, so machte sich eine deutliche Erwärmung bemerkbar. Nach längerem Kochen am Rückflusskühler wurde die Aetherlösung abfiltrirt, und der Aether abdestillirt. Der hierbei zurückbleibende Rückstand wurde dann aus leicht flüchtigem Petrolenmäther umkrystallisirt, wobei derbe, glänzende Prismen vom Schmelzp. $76^{\circ}$ erhalten wurden.

Die Brombestimmung ergab:

$0,169 \mathrm{Grm}$. Substanz lieferten 0,208 Grm. AgBr oder 52,07\% Br.

$0,203 \mathrm{Grm}$. Substanz lieferten 0,250 Grm. AgBr oder 52,21\% $\mathrm{Br}$.

Die Formel $\mathrm{C}_{10} \mathrm{H}_{10} \mathrm{Br}_{2} \mathrm{O}$ verlangt 52,28\% $\mathrm{Br}$.

Die Verbindung entsprach somit einem Dibromanethol, $\mathrm{C}_{6} \mathrm{H}_{2} \mathrm{Br}_{2}$. $\mathrm{CH}=\mathrm{CH} . \mathrm{CH}_{3}$, isomer mit der durch Behandeln des Monobromanetholbromids mit siedendem Alkol erhaltenen Verbindung $\mathrm{C}_{6} \mathrm{H}_{3} \mathrm{Br}\left(\mathrm{OCH}_{3}\right) \cdot \mathrm{CBr}=\mathrm{CH} . \mathrm{CH}_{3}$. Beim Uebergiessen mit Brom wird das letztere lebhaft und ohne $\mathrm{BrH}$-Entwicklung aufgenommen, indem das ursprüngliche bei $113^{\circ}$ schmelzende Dibromanetholdibromid, $\mathrm{C}_{10} \mathrm{H}_{10} \mathrm{Br}_{4} \mathrm{O}$, wieder zurückgebildet wird. Es ist dieses Verhalten insofern bemerkenswerth, als sich dem Monobromanetholdibromid bei analoger Behandlung auch zwei Bromatome entziehen lassen; das entstandene Produkt sich aber polymerisirt, und sich dem zu Folge nicht mehr mit Brom verbindet.

Aus diesem Reductionsversuch scheint ferner hervor zugehen, dass bei der weiteren Bromirung des Monobromanetholdibromids das 4. Bromatom ebenfalls in den Kern tritt.

Einwirkung von Anilin auf das Dibromanetholdibromid.

Wurde das Dibromanetholdibromid mit überschüssigem Anilin zusammengebracht, so trat unter schwacher Erwärmung eine vollständige Lösung ein und beim Erhitzen entstand eine lebhafte Reaction, indem sich bromwasserstoffsaures 
Hell u. v. Günthert: Beitr. z. Kenntn. des Anethols. 205

Anilin abschied. Dasselbe wurde abfiltrirt, und die Anilinlösung mit Salzsäure versetzt, wobei ein gelbliches Pulver resultirte, welches nicht krystallisirt erhalten werden konnte und sich leicht in Aether, Alkohol und Benzol löste, beim Verdunsten der Lösungsmittel aber als nicht mehr erstarrendes Harz zurückblieb. Das feste, durch Versetzen seiner Anilinlösung mit Salzsäure erhaltene Produkt wies einen Schmelzp. von $82^{\circ}$ auf.

Die Bestimmung seines Bromgehaltes ergab:

0,316 Grm. Substanz gaben 0,300 Grm. AgBr oder $40,18 \% \mathrm{Br}$.

Berechnet für die Formel $\mathrm{C}_{10} \mathrm{H}_{16} \mathrm{OBr}_{2} \mathrm{~N} 40,3 \% \mathrm{Br}$.

Die Constitution dieser Verbindung entspricht somit wahrscheinlich der Formel:

$$
\mathrm{C}_{8} \mathrm{H}_{2} \mathrm{Br}_{8}\left(\mathrm{OCH}_{3}\right) \mathrm{CH} . \mathrm{CH} . \mathrm{CH}_{3} \text {. }
$$

\section{Oxydation des Dibromanetholdibromids mit Chromsäure.}

Beim Eintragen von $10 \mathrm{Grm}$. des Tetrabromids in die eisessigsaure Lösung von Chromsäure (2 Mol.) findet schon in der Kälte eine sehr lebhafte Reaction unter Bromentwicklung statt, und beim Erwärmen ist die Oxydation schon nach kurzer Zeit beendet. Nach dem Abdestilliren des Eisessigs wird die Masse in Wasser gegossen und mit Sodalösung ausgekocht, wodurch eine kleine Menge von Säuren, von denen später die Rede sein soll, aufgenommen wurde.

Der in Natriumcarbonat unlösliche Theil des Oxydationsproduktes wurde in Aether gelöst und daraus durch Alkohol gefällt. Man erbielt so ein gelblich gefärbtes, amorphes Pulver, welches sich leicht in Aether, Benzol und Chloroform, schwer in Alkohol und Eisessig löste und ganz unlöslich in Petroleumäther war. Beim Verdunsten dieser Lösungsmittel blieb es auch wieder als gelbes Pulver zurück, welches keine Andeutung von krystallinischer Struktur zeigte. Der Schmelzp. lag bei $135^{\circ}$.

Bei der Analyse wurde gefunden:

1. 0,397 Grm. Substanz gaben 0,433 Grm. $\mathrm{CO}_{2}$ und $0,061 \mathrm{Grm} . \mathrm{H}_{2} \mathrm{O}$. $0,325 \mathrm{Grm}$. Substanz gaben $0,450 \mathrm{Grm}$. AgBr.

2. 0,429 Grm. Substanz gaben 0,467 Grm. $\mathrm{CO}_{2}$ und $0,082 \mathrm{Grm}$. $\mathrm{H}_{2} \mathrm{O}$. $0,322 \mathrm{Grm}$. Substanz gaben $0,445 \mathrm{Grm}$. AgBr. 
206 Hell u. v. Günthert: Beitr. z. Kenntn. des Anethols.

\begin{tabular}{lcrr} 
& Berechnet für & \multicolumn{2}{c}{ Gefunden : } \\
& $\mathrm{C}_{10} \mathrm{H}_{9} \mathrm{Br}_{3} \mathrm{O}_{2}:$ & 1. & 2. \\
$\mathrm{C}$ & 29,9 & 29,72 & $29,60 \%$ \\
$\mathrm{H}$ & 2,2 & 1,70 & $2,12 \%$ \\
$\mathrm{Br}$ & 59,8 & 58,76 & $58,69 \%$
\end{tabular}

Der beide Male etwas zu niedrig gefundene Bromgehalt erklärt sich dadurch, dass die Zerstörung der Substanz durch Salpetersäure in der Carius'schen Röhre ausserordentlich schwer vor sich geht.

Die Einwirkung der Chromsäure auf das höhere Anetholbromid hat somit gleichfalls in der bei dem Anetholbromid und seinem Monobromsubstitutionsprodukt beobachteten Weise stattgefunden. Es ist ein Keton, $\mathrm{C}_{10} \mathrm{H}_{9} \mathrm{Br}_{3} \mathrm{O}_{2}$, entstanden, in welchem noch 3 Atome Brom, und zwar, wie sich aus den später mitzutheilenden Versuchen ergiebt, 2 im aromatischen Kern und 1 in der Seitenkette vorhanden sind.

In der zum Auskochen des rohen Oxydationsproduktes benutzten Sodalösung fanden sich gebromte Säuren vor. Beim Fällen mit Salzsäure wurde ein dunkel gefärbter Niederschlag erhalten, welcher auch nach wiederholtem Auflösen in Alkalien und Fällen mit Säuren ein dunkles Aussehen besass und der bei $210^{\circ}$ noch nicht geschmolzen war. Wahrscheinlich liegt hier eine Dibromanissäure vor. Die von dieser Säure abfiltrirte Lösung gab an Aether eine zweite Säure ab, die beim Verdunsten als ein rothbrauner, harziger Körper zurückblieb, der sich in Wasser ziemlich leicht mit weinrother Farbe löste und beim Verdunsten der wässrigen Lösung in feinen Nadeln krystallisirte. Durch wiederholtes Umkrystallisiren konnten dieselben vollständig farblos und rein erhalten werden. Ihr Schmelzpunkt lag bei $85^{\circ}$. Sie lösten sich leicht in Ammoniak und Alkalien, ebenso in Wasser. Leider war die Menge so unbedeutend, dass nur eine Brombestimmung damit ausgeführt werden konnte.

0,243 Grm. Substanz gaben 0,260 Grm. AgBr oder 45,26\% Br, was auf eine Säure $\mathrm{C}_{10} \mathrm{H}_{8} \mathrm{Br}_{2} \mathrm{O}_{4}$, welche $45,4 \%$ Brom verlangt, schliessen lässt.

Wir enthalten uns daher, ehe wir eine eingehendere Untersuchung mit neuem Material vornehmen können, über die Natur dieser Säure weitere Betrachtungen anzustellen. 
Hell u. v. Günthert: Beitr. z. Kenntn. des Anethols. 207

Einwirkung von Ammoniak auf das Keton $\mathrm{C}_{10} \mathrm{H}_{9} \mathrm{Br}_{3} \mathrm{O}_{2}$. Beim Erhitzen mit überschüssigem alkoholischen Ammoniak im zugeschmolzenen Rohr im Wasserbade findet keine Einwirkung statt. Eine solche tritt erst ein, wenn die Röhre auf $180^{\circ}$ erhitzt wird. Es resultirt nach dem Verdunsten des Alkohols und Ammoniaks ein bräunlich aussehender Körper von amorpher Beschaffenheit, der sich in den gewöhnlichen Lösungsmitteln wie Aether, Alkohol, Benzol, Petroleumäther, Chloroform und Schwefelkohlenstoff nicht löste. Ebenso unlöslich war er auch in verdünnten Säuren und in Wasser. Zum Schmelzen konnte er nicht gebracht werden, sondern sublimirte bei sehr hoher Temperatur. Auf dem Platinblech verbrannte diese Verbindung obne Rückstand. Beim Kochen mit conc. Salpetersäure bildete sich ein röthlicher Körper, welcher beim Erhitzen lebhaft verpuffte. In conc. Schwefelsäure löst das Produkt sich auf und fällt auf Zusatz von Wasser unverändert wieder aus.

Die Bestimmung des Bromgehalts ergab:

0,276 Grm. Substanz lieferten $0,322 \mathrm{Grm}$. AgBr oder $49,63 \% \mathrm{Br}$.

0,289 Grm. Substanz lieferten 0,340 Grm. AgBr ader $49,82 \% \mathrm{Br}$.

Die Formel $\mathrm{C}_{10} \mathrm{H}_{9} \mathrm{Br}_{2} \mathrm{NO}$ verlangt $50,1 \% \mathrm{Br}$.

Es scheint somit, dass die Einwirkung des Ammoniaks analog derjenigen ist, wie sie auf die bromärmeren Ketone vor sich gegangen ist. Der Unterschied liegt hauptsächlich in der Temperatur. Während bei dem Mono- und Dibromketon das Ammoniak schon bei Wasserbadtemperatur einwirkt, findet bei dem. Tribromketon die Einwirkung erst bei höherer Temperatur statt. Die Formel für das Ammoniakprodukt wäre danach durch

$$
\mathrm{C}_{6} \mathrm{H}_{2} \mathrm{Br}_{2}\left(\mathrm{OCH}_{3}\right) \cdot \mathrm{CH}-\mathrm{C} . \mathrm{CH}_{8} \text { oder } \mathrm{C}_{6} \mathrm{H}_{2} \mathrm{Br}_{2}\left(\mathrm{OCH}_{3}\right) \cdot \mathrm{C}-\mathrm{CH} . \mathrm{CH}_{3} \text {, }
$$

resp. durch Verdoppelung derselben auszudrücken.

Einwirkung von Anilin auf das Keton.

Von hervorragendem Interesse ist die Einwirkung des Anilins auf das dreifach bromirte Keton. Erhitzt man dasselbe mit einer alkoholischen Anilinlösung längere Zeit am Rückflusskühler, so bildet sich neben bromwasserstoffsaurem Anilin ein chokoladefarbiger, anscheinend amorpher Körper, der sich schwer in Alkohol, leichter in Aether und Benzol 
208 Hell u. v. Günthert: Beitr. z. Kenntn. des Anethols.

löste, aus diesen Lösungsmitteln aber stets amorph sich wieder abschied. Wird derselbe jedoch in siedendem Anilin aufgelöst, so scheiden sich nach dem Erkalten der Lösung prachtvolle, bronzefarbene, glänzende Blättchen ab, welche sich leicht durch Absaugen und Auswaschen mit verdünnter Salzsäure von der überschüssigen Anilinlösung befreien lassen. Denselben Körper von gleichem Aussehen erhält man auch, wenn man das Keton in überschüssigem siedenden Anilin auflöst. Aus der abgesaugten Anilinlösung lässt sich noch mehr von dieser Verbindung erhalten, wenn man Alkohol hinzufügt, nur scheidet sich dieselbe dann nicht mehr krystallisirt, sondern, wie schon oben beschrieben, als amorphes, chokoladebraunes Pulver ab, das aber durch Aufösen in siedendem Anilin wieder leicht krystallisirt erhalten werden kann. Auf dem Platinblech erhitzt, entwickelt das Produkt einen intensiv gelb gefärbten Dampf von eigenthümlichem, nicht unangenehmem. aromatischen Geruch und verbrennt zuletzt ohne jeglichen Rückstand. In conc. Schwefelsäure löst es sich mit prachtvoller, carmoisinrother Färbung auf und auf Zusatz von Wasser scheidet sich wieder der ursprüngliche amorphe Körper pulverförmig ab. Auch in conc. Salpetersäure findet theilweise Auflösung unter Rothfärbung statt, von conc. Salzsäure wird dagegen die Verbindung nicht angegriffen.

AuffallenderWeise enthielt dieseVerbindung kein Brom mehr, trotz der beiden im Benzolkern noch anzunehmenden Bromatome.

Bei der Elementaranalyse wurden folgende Werthe erhalten:

$0,147 \mathrm{Grm}$. bei $120^{\circ}$ getrockneter Substanz gaben 0,417 Grm. CO. und 0,089 Grm. $\mathrm{H}_{2} \mathrm{O}$.

$0,213 \mathrm{Grm}$. Substanz gaben $18,0 \mathrm{Ccm}$. $\mathbf{N}$ bei $16^{\circ}$ und $730 \mathrm{Mm}$.

$\begin{array}{lc}\mathrm{C} & \mathbf{7 6 , 8 7} \% \\ \mathrm{H} & 6,7 \% \\ \mathrm{~N} & 9,61 \% .\end{array}$

Diese analytischen Werthe lassen zwei verschiedene Deutungen zu. Die Formel $\mathrm{C}_{28} \mathrm{H}_{27} \mathrm{O}_{2} \mathrm{~N}_{3}$, entstanden nach der Gleichung: $\begin{aligned} \mathrm{C}_{10} \mathrm{H}_{6} \mathrm{Br}_{3} \mathrm{O}_{2}+6 \mathrm{NH}_{2} \mathrm{C}_{6} \mathrm{H}_{5}= & \mathrm{C}_{6} \mathrm{H}_{2}\left(\mathrm{NHC}_{6} \mathrm{H}_{5}\right)_{2}\left(\mathrm{OCH}_{3}\right) \\ & \text { COCH}\left(\mathrm{NHC}_{6} \mathrm{H}_{6}\right) \cdot \mathrm{CH}_{8}+3 \mathrm{C}_{6} \mathrm{H}_{5} \mathrm{NH}_{2} \cdot \mathrm{HBr} \text {, }\end{aligned}$ verlangt

$\begin{array}{cc}\mathrm{C} & 76,9 \% \\ \mathrm{H} & 6,1 \% \\ \mathrm{~N} & 9,8,,\end{array}$


Hell u. v. Günthert: Beitr. z. Kenntn. des Anethols. 209

während die Formel $\mathrm{C}_{50} \mathrm{H}_{47} \mathrm{O}_{4} \mathrm{~N}_{5}$, welche man nach der Gleichung:

$2 \mathrm{C}_{10} \mathrm{H}_{9} \mathrm{Br}_{3} \mathrm{O}_{2}+11 \mathrm{NH}_{2} \mathrm{C}_{6} \mathrm{H}_{5}=\mathrm{C}_{6} \mathrm{H}_{2}\left(\mathrm{NHC}_{6} \mathrm{H}_{5}\right)_{2}\left(\mathrm{OCH}_{3}\right)$. CO. CH. $\mathrm{CH}_{8}$

$$
\mathrm{C}_{6} \mathrm{H}_{2}\left(\mathrm{NHC}_{6} \mathrm{H}_{6}\right)_{2}\left(\mathrm{OCH}_{8}\right) \text {. CO. CH. } \mathrm{CH}_{3}
$$

sich entstanden denken kann,

verlangt.

$\begin{array}{lr}\mathrm{C} & \mathbf{7 6 , 8 \%} \\ \mathrm{H} & 6,0 " \\ \mathrm{~N} & \mathbf{8 , 9} \%\end{array}$

Die Procentzahlen dieser beiden Formeln kommen, besonders was den Kohlenstoff- und Wasserstoffgehalt betrifft, einander so nahe, dass eine Entscheidung durch die Analyse nicht wohl möglich ist. Grössere Wahrscheinlichkeit hat jedoch erstere Formel.

Soviel steht jedenfalls fest, dass das Anilin nicht nur das Bromatom der Seitenkette, sondern sämmtliche Bromatome des Moleküls zum Austritt gezwungen hat.

Bemerkenswerth ist der Unterschied, welcher zwischen der Einwirkung des Anilins auf das Dibromanetholdibromid und das daraus durch Oxydation gewonnene Keton $\mathrm{C}_{10} \mathrm{H}_{9} \mathrm{Br}_{3} \mathrm{O}_{2}$ besteht. Während in dem ersten Falle bei der Einwirkung des Anilins noch $z$ wei Bromatome unangegriffen bleiben, werden bei dem Keton die sämmtlichen Bromatome durch das Anilin eliminirt.

Oxydation des Ketons mit Kaliumpermanganat.

Schliesslich führte ich noch mit der übrig gebliebenen kleinen Menge des Ketons einen Oxydationsversuch mit Chamäleonlösung aus. Das Kaliumpermanganat wirkt ungleich schwerer auf das Tribromketon, als auf die entsprechenden vorhergehenden bromärmeren Ketone ein. Es bedurfte tagelangen Kochens mit der oxydirenden Lösung, um nur wenige Gramm des Ketons zu oxydiren. Nachdem das entstandene Mangansuperoxyd abfiltrirt war, wurde die alkalisch reagirende Lösung eingedampft und hierauf mit Schwefelsäure versetzt. Es entstand eine Trübung, wahrscheinlich von der Bildung einer kleinen Menge von Dibromanissäure herrübrend, die jedoch zu gering war, um eine genaue Untersuchung zu gestatten.

Journal f. prakt. Chemie [2] Bd. bz. 
210 Cartius: Ueb. Benzazide u. einige Säureazide d. Fettr.

Nach dem Abdestilliren der Lösung konnte im Destillat Essigsäure kaum nachgewiesen werden, dagegen war es möglich, durch Ausschütteln mit Aether einen kleinen Theil einer Verbindung zu erhalten, welche sich ziemlich leicht in Wasser löste und daraus in kleinen, feinen Nadeln vom Schmelzp. $85^{\circ}$ krystallisirte und daher ohne Zweifel mit der auf S. 206 beschriebenen, bei der Oxydation des Dibromanetholdibromids mit Chromsäure als Nebenprodukt erhaltenen Säure, vielleicht eine Dibromketonsäure $\mathrm{C}_{10} \mathrm{H}_{3} \mathrm{Br}_{2} \mathrm{O}_{4}($ ? $)$, identisch war. In den Mangansuperoxydniederschlägen befand sich noch ein erheblicher Theil unoxydirt gebliebenen Ketons, welcher durch Extrahiren mit Aether wiedergewonnen werden konnte.

Ueber weitere Bromderivate des Anethols, insbesondere über die durch Einwirkung von Brom auf die gebromten Ketone resultirenden Verbindungen werden weitere Mittheilungen folgen.

Stuttgart, technische Hochschule, Laboratorium für allgemeine Chemie.

\title{
Mittheilungen aus dem chemischen Institute der Universität Kiel.
}

\author{
Hydrazide und Azide organischer Sänren; \\ ron
}

Th. Gurtius.

VII. Abhandlung.

31. Ueber Benzazid und einige Sänreazide der Fettreihe ${ }^{1}$ ); von

Th. Curtius.

Die Darstellung des Benzazids $\mathrm{O}_{6} \mathrm{H}_{5} \mathrm{CON}_{3}$ ist schon beschrieben worden (XXIIb). ${ }^{2}$ ) Zur Charakteristik füge ich noch folgendes hinzu. Um die Substanz ganz rein zu erhalten, nimmt man dieselbe am besten mit Aceton auf, trocknet über Chlor-

1) Vergl. dies. Journ. [2] 50, 285-294. Die römischen Zahlen entsprechen ebensolchen in der Abhandlung $I_{\text {, ebenda S. } 275 .}$

2) Ber. 23, 3029. 